\title{
RE-EXAMINATION OF TWO ZERO TEXTURE OF NEUTRINO MASS MATRIX AFTER NEUTRINO-2012
}

\author{
NGUYEN THANH PHONG \\ Department of Physics, Can Tho University, Can Tho city, Can Tho, Vietnam \\ Email: thanhphong@ctu.edu.vn \\ Received 14 August 2013; \\ Accepted for publication 28 August 2013
}

\begin{abstract}
We re-study the seven neutrino mass matrices with two independent vanishing entries under the latest results of neutrino oscillation experiments after the neutrino - 2012 conference. It is shown that the pattern $B_{1}$ could be excluded since this pattern realizes very small value of mixing angle $\theta_{13}$. The remain six textures satisfy the current experimental data. We also find that all seven textures of neutrino mass matrix possess the normal hierarchy of neutrino mass spectrum.
\end{abstract}

\section{INTRODUCTION}

The evidences of the oscillations of neutrino has been dramatically confirmed by a numerous neutrino experiments from different sources: solar, atmospheric, reactor and accelerator neutrino after Super-Kamiokande [1-3]. Based on the neutrino experimental results, Frampton et al. [4] found seven acceptable patterns of the neutrino mass matrix with two independent vanishing components in the basis where the charged lepton mass matrix is diagonal. Subsequently, these patterns have been considerably studied by many authors, see for examples [5-7] and references therein, and recently in $[8,9]$. Besides, since Frampton's seven patterns was not explained by any mechanism, therefore Kageyama et al. [7] proposed the seesaw mechanism to generate these textures of neutrino mass matrix.

After the neutrino - 2012 conference [10], based on the recent results of T2K [11], MINOS [12], RENO [13], Double CHOOZ [14] and Daya Bay [15] experiments, the newest values of lepton mixing angles are established where the reactor mixing angle $\theta_{13}$ is rather large. It motivates for model builders to find best neutrino mass model that well described the latest neutrino experimental results. Therefore, it is interesting to re-evaluate the neutrino mass matrices with two independent vanishing components, one of the well known simple neutrino mass model introduced in [4]. Actually, these textures was re-evaluated by Fritzsch et al. [8] after the early results of T2K [11] and MINOS [12] and then later by Liu et al. [9] after RENO [13] and Daya Bay [15]. However, our approach is a little different. The authors in $[8,9]$ find the relations among low energy observables $\left(m_{1,2,3}, \theta_{12,13,23}, \mathrm{CP}\right.$ violating phases) and using the experimental data to evaluate the textures. Our method is finding the allowed values of the parameters of the aforementioned seven patterns of 
neutrino mass matrix with two independent vanishing entries based on the the global fit of neutrino experimental data after the neutrino - 2012 meeting, done by Forero et al. [10].

The remain of this work is organized as followed. We first diagonalize the Hermitian matrix $m_{\nu}^{\dagger} m_{\nu}$ to find squared neutrino masses, neutrino mixing angles and Dirac CP violating phase in terms of the components of neutrino mass matrices in the next section. Sec. III is devoted to show our numerical calculations based on the analysis formulae in Sec. II and experimental data from the reference [10]. Our conclusions are given in the last section.

\section{NEUTRINO MASS MATRICES WITH TWO ZERO ENTRIES}

As mentioned above, there are seven acceptable textures with two independent zeros for the neutrino mass matrix $m_{\nu}$ which classified in to three categories [4]

$$
\begin{aligned}
& \mathbf{A}_{1}: m_{\nu}=v c\left(\begin{array}{ccc}
0 & 0 & e^{i \varphi_{1}} \\
0 & \kappa & \rho e^{i \varphi_{2}} \\
e^{i \varphi_{1}} & \rho e^{i \varphi_{2}} & \chi
\end{array}\right) ; \mathbf{A}_{2}: m_{\nu}=v c\left(\begin{array}{ccc}
0 & e^{i \varphi_{1}} & 0 \\
e^{i \varphi_{1}} & \kappa & \rho e^{i \varphi_{2}} \\
0 & \rho e^{i \varphi_{2}} & \chi
\end{array}\right) \\
& \mathbf{B}_{1}: m_{\nu}=v c\left(\begin{array}{ccc}
\kappa & e^{i \varphi_{1}} & 0 \\
e^{i \varphi_{1}} & 0 & \rho e^{i \varphi_{2}} \\
0 & \rho e^{i \varphi_{2}} & \chi
\end{array}\right) ; \mathbf{B}_{2}: m_{\nu}=v c\left(\begin{array}{ccc}
\kappa & 0 & \rho e^{i \varphi_{1}} \\
0 & \chi & e^{i \varphi_{2}} \\
\rho e^{i \varphi_{1}} & e^{i \varphi_{2}} & 0
\end{array}\right) ; \\
& \mathbf{B}_{3}: m_{\nu}=v c\left(\begin{array}{ccc}
\kappa & 0 & e^{i \varphi_{1}} \\
0 & 0 & \rho e^{i \varphi_{2}} \\
e^{i \varphi_{1}} & \rho e^{i \varphi_{2}} & \chi
\end{array}\right) ; \mathbf{B}_{4}: m_{\nu}=v c\left(\begin{array}{ccc}
\kappa & e^{i \varphi_{1}} & 0 \\
e^{i \varphi_{1}} & \chi & \rho e^{i \varphi_{2}} \\
0 & \rho e^{i \varphi_{2}} & 0
\end{array}\right) \\
& \mathbf{C}: m_{\nu}=v c\left(\begin{array}{ccc}
\chi & e^{i \varphi_{1}} & \kappa e^{i \varphi_{3}} \\
e^{i \varphi_{1}} & 0 & \rho e^{i \varphi_{2}} \\
\kappa e^{i \varphi_{3}} & \rho e^{i \varphi_{2}} & 0
\end{array}\right) \text {, }
\end{aligned}
$$

where $v=174 \mathrm{GeV}$ is the VEV of Standard Model's Higgs, and $c$ is the global parameter which can be roughly estimated by $c \simeq \frac{\sqrt{\left|\Delta m_{31}^{2}\right|}}{v}$. Here we suppose that $c$ and diagonal parameters are all real.

Before discussing if these patterns are consistent with the current neutrino experimental results, we first diagonalize the neutrino mass matrix by $U_{\text {PMNS }}$ as

$$
U_{\mathrm{PMNS}}^{T} m_{\nu} U_{\mathrm{PMNS}}=\text { Diag. }\left(m_{\nu}\right)=\left(\begin{array}{ccc}
m_{1} & 0 & 0 \\
0 & m_{2} & 0 \\
0 & 0 & m_{3}
\end{array}\right) .
$$

where $U_{\mathrm{PMNS}}$ is the neutrino mixing matrix which parameterized as $U_{\mathrm{PMNS}}=U_{\nu} K$ with

$$
\begin{aligned}
U_{\nu} & =\left(\begin{array}{ccc}
c_{12} c_{13} & s_{12} c_{13} & s_{13} e^{-i \delta_{\mathrm{CP}}} \\
-c_{23} s_{12}-s_{23} c_{12} s_{13} e^{i \delta_{\mathrm{CP}}} & c_{23} c_{12}-s_{23} s_{12} s_{13} e^{i \delta_{\mathrm{CP}}} & s_{23} c_{13} \\
s_{23} s_{12}-c_{23} c_{12} s_{13} e^{i \delta_{\mathrm{CP}}} & -s_{23} c_{12}-c_{23} s_{12} s_{13} e^{i \delta_{\mathrm{CP}}} & c_{23} c_{13}
\end{array}\right), \\
K & =\operatorname{diag}\left(e^{i \rho}, e^{i \sigma}, 1\right),
\end{aligned}
$$

where $c_{i j}=\cos \theta_{i j}, s_{i j}=\sin \theta_{i j}(i j=12,23,13), \delta_{\mathrm{CP}}$ is Dirac CP violating phase and $\rho, \sigma$ are two Majorana $\mathrm{CP}$ violating phases. However, instead of diagonalize $m_{\nu}$, we diagonalize the Hermitian matrix $m_{\nu}^{\dagger} m_{\nu}$ to examine the structure of $m_{\nu}$, so that the two Majorana 
phases become relevant [16]. To do so, we set the seven patterns of $m_{\nu}$ in eqs. $(1,2,3)$ in a general form as

$$
m_{\nu}=\left(\begin{array}{lll}
a & b & c \\
b & d & e \\
c & e & f
\end{array}\right)
$$

so that we can easy obtain the squared neutrino masses, the mixing angles and Dirac CP phase in terms of the parameters appeared in $m_{\nu}$

$$
m_{\nu}^{\dagger} m_{\nu}=U_{\mathrm{PMNS}} \operatorname{Diag}\left(m_{1}^{2}, m_{2}^{2}, m_{3}^{2}\right) U_{\mathrm{PMNS}}^{\dagger} \equiv\left(\begin{array}{ccc}
A & \tilde{B} & \tilde{C} \\
\tilde{B}^{*} & D & \tilde{E} \\
\tilde{C}^{*} & \tilde{E}^{*} & F
\end{array}\right)
$$

where

$$
\begin{aligned}
& A=|a|^{2}+|b|^{2}+|c|^{2}, \quad \tilde{B}=a^{*} b+b^{*} d+c^{*} e, \\
& \tilde{C}=a^{*} c+b^{*} e+c^{*} f, \quad D=|b|^{2}+|d|^{2}+|e|^{2}, \\
& \tilde{E}=b^{*} c+d^{*} e+e^{*} f, \quad F=|c|^{2}+|e|^{2}+|f|^{2} .
\end{aligned}
$$

Then, the straightforward calculation with the standard parametrization of $U_{\text {PMNS }}$ leads to the expressions for the masses and mixing parameters

$$
\begin{aligned}
& m_{1,2}^{2}=\frac{\lambda_{1}+\lambda_{2}}{2} \mp \frac{c_{23} \operatorname{Re}(\tilde{B})-s_{23} \operatorname{Re}(\tilde{C})}{2 s_{12} c_{12} c_{13}} ; \\
& m_{3}^{2}=\frac{c_{13}^{2} \lambda_{3}-s_{13}^{2} A}{c_{13}^{2}-s_{13}^{2}}, \\
& \tan \theta_{23}=\frac{\operatorname{Im}(\tilde{B})}{\operatorname{Im}(\tilde{C})} ; \tan 2 \theta_{12}=2 \frac{c_{23} \operatorname{Re}(\tilde{B})-s_{23} \operatorname{Re}(\tilde{C})}{c_{13}\left(\lambda_{2}-\lambda_{1}\right)} ; \\
& \tan 2 \theta_{13}=2 \frac{\left|s_{23} \tilde{B}+c_{23} \tilde{C}\right|}{\lambda_{3}-A}, \\
& \tan \delta_{\mathrm{CP}}=-\frac{1}{s_{23}} \frac{\operatorname{Im}(\tilde{B})}{s_{23} \operatorname{Re}(\tilde{B})+c_{23} \operatorname{Re}(\tilde{C})},
\end{aligned}
$$

with

$$
\begin{aligned}
& \lambda_{1}=c_{13}^{2} A-2 s_{13} c_{13}\left|s_{23} \tilde{B}+c_{23} \tilde{C}\right|+s_{13}^{2} \lambda_{3}, \\
& \lambda_{2}=c_{23}^{2} D+s_{23}^{2} F-2 s_{23} c_{23} \operatorname{Re}(\tilde{E}), \\
& \lambda_{3}=s_{23}^{2} D+c_{23}^{2} F+2 s_{23} c_{23} \operatorname{Re}(\tilde{E}) .
\end{aligned}
$$

As can be seen from Eqs. (10,11,12,13), three neutrino masses, three mixing angles and a $\mathrm{CP}$ phase are presented in terms of five independent parameter $\chi, \kappa, \rho, \varphi_{1}, \varphi_{2}$ and addition phase $\varphi_{3}$ only for the pattern $\mathbf{C}$. At the present, we have five experimental results, which are taken as inputs in our numerical analysis given at $3 \sigma$ by [10]

$$
\begin{aligned}
& 31.3^{0} \leq \theta_{12} \leq 37.5^{0}, 37^{0} \leq \theta_{23} \leq 55.5^{0}, 7.5^{0} \leq \theta_{13} \leq 10.5^{0} \\
& 7.12 \leq \Delta m_{21}^{2}\left[10^{-5} \mathrm{eV}^{2}\right] \leq 8.20,2.31(2.21) \leq\left|\Delta m_{31}^{2}\right|\left[10^{-3} \mathrm{eV}^{2}\right] \leq 2.74(2.64),
\end{aligned}
$$


where numbers inside the parentheses correspond to the inverted hierarchy of neutrino masses. And the Dirac CP violating phase, $\delta_{\mathrm{CP}}$, is unrestricted at $3 \sigma$ confident level.

\section{NUMERICAL RESULTS}

The numerical calculations are done in the following way:

(1) Using the current experimental results on neutrino masses and mixing angles in Eq. (14) for the case of normal hierarchy of light neutrino masses, except that for the reactor mixing angle we have used $0^{0} \leq \theta_{13} \leq 11^{0}$.

(2) Three phases $\varphi_{1}, \varphi_{2}, \varphi_{3}$ have a random value from zero to $2 \pi$.

(3) Imposing the curent experimental results on the neutrino masses, mixing angles into the above relations [Eqs. $(10,11,12,13)$ ], scanning all the allowed parameter space $\chi, \kappa, \rho, \varphi_{1}, \varphi_{2}, \varphi_{3}$. We investigate how those parameters are constrained and estimate possible prediction for Dirac $\mathrm{CP}$ violating phase $\delta_{\mathrm{CP}}$ and the mixing angle $\theta_{13}$.

Our numerical results are plotted in figures $1 \div 7$ corresponding to the seven patterns. In each figure, the top panels show the allowed parameter space, whereas the bottom panels present the predictions of the Dirac CP violating phase (left panels) and the mixing angle $\theta_{13}$ (right panels).
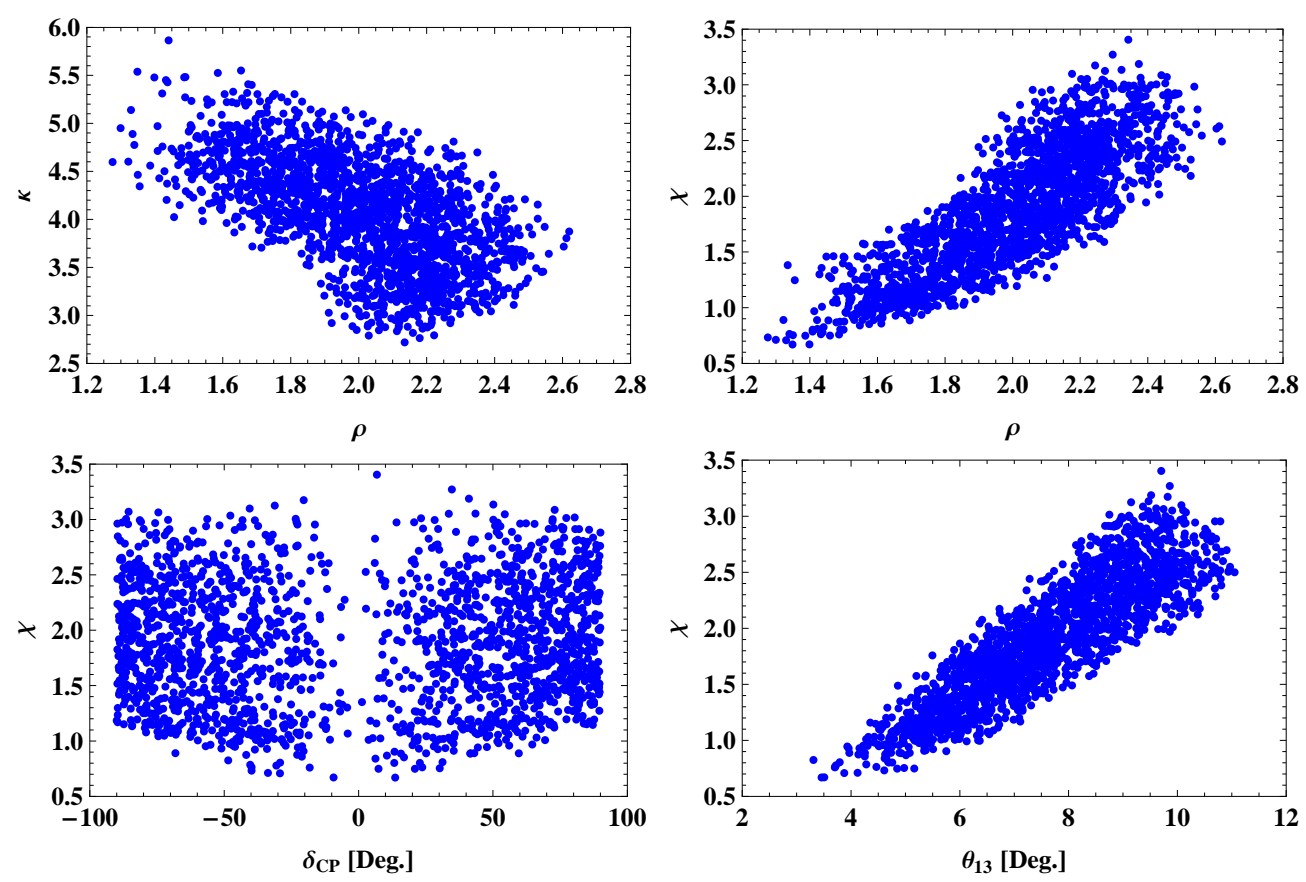

Fig. 1. The allowed parameter $\kappa, \rho, \chi$ (top panels) and the predictions of the Dirac $\mathrm{CP}$ violating phase $\delta_{\mathrm{CP}}$ (bottom-left panel) and the mixing angle $\theta_{13}$ (bottom-right panel) of the pattern $A_{1}$. 

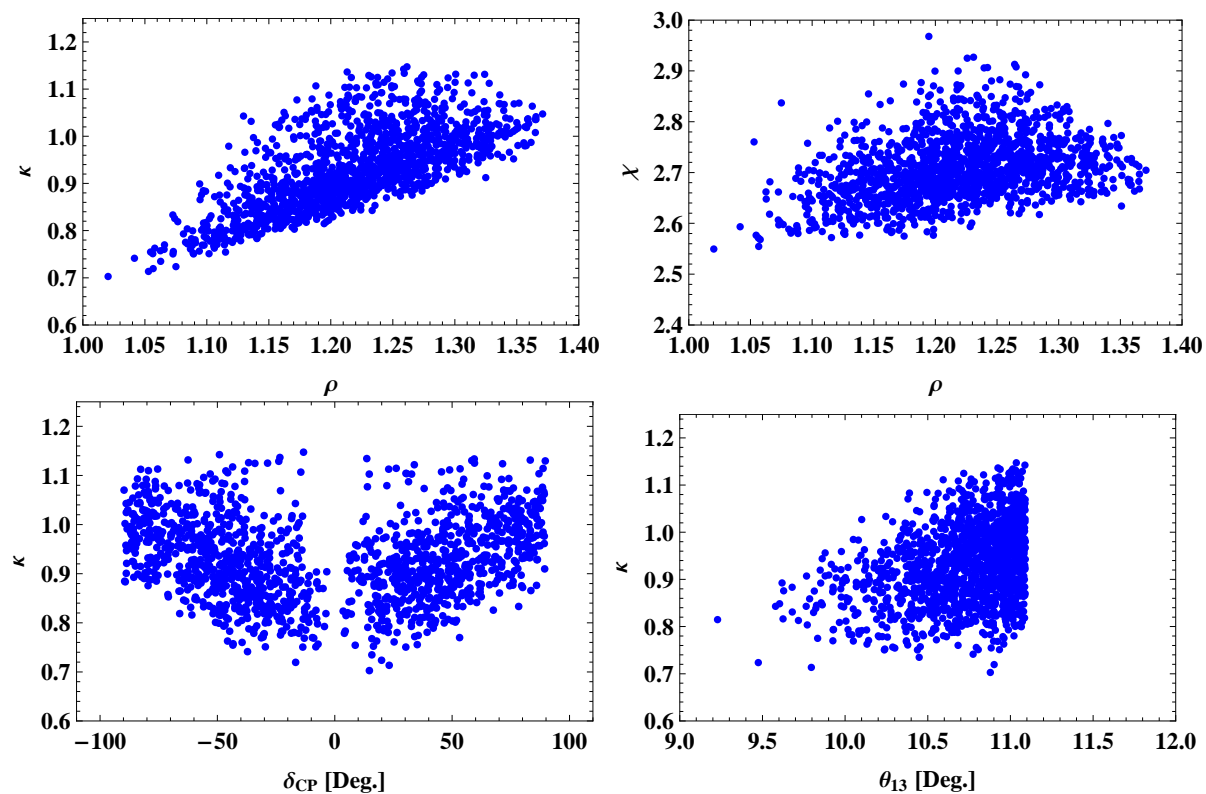

Fig. 2. The allowed parameter $\kappa, \rho, \chi$ (top panels) and the predictions of the Dirac $\mathrm{CP}$ violating phase $\delta_{\mathrm{CP}}$ (bottom-left panel) and the mixing angle $\theta_{13}$ (bottom-right panel) of the pattern $\mathrm{A}_{2}$.
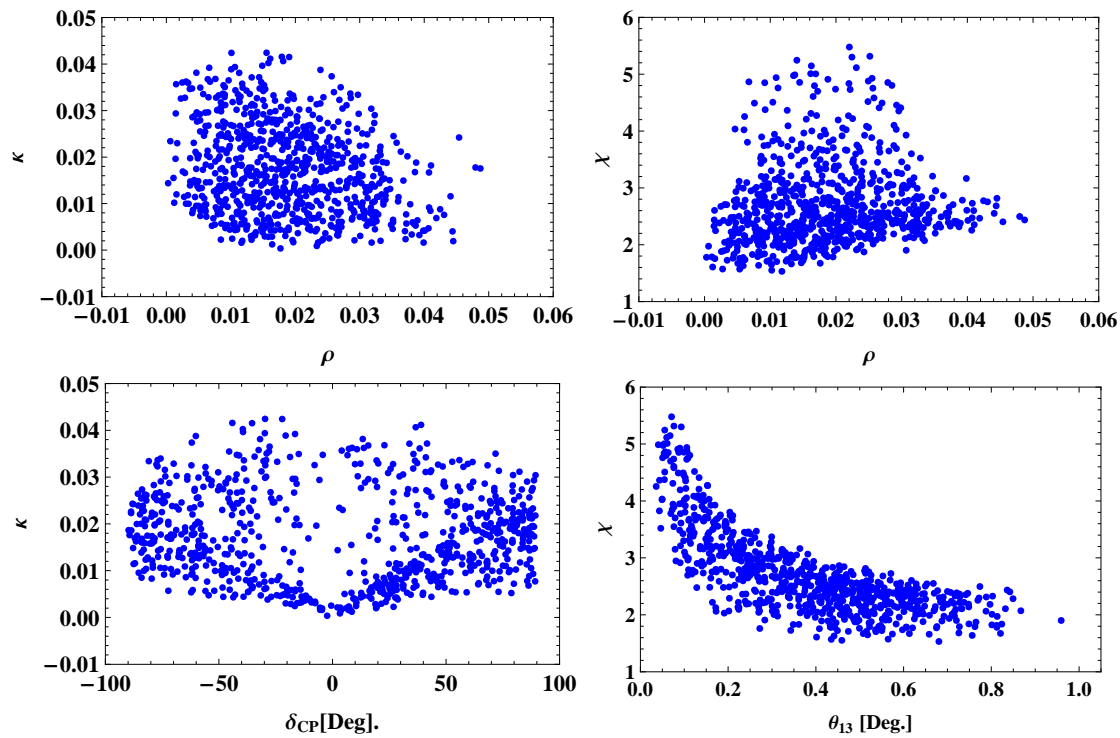

Fig. 3. The allowed parameter $\kappa, \rho, \chi$ (top panels) and the predictions of the Dirac $\mathrm{CP}$ violating phase $\delta_{\mathrm{CP}}$ (bottom-left panel) and the mixing angle $\theta_{13}$ (bottom-right panel) of the pattern $B_{1}$. 

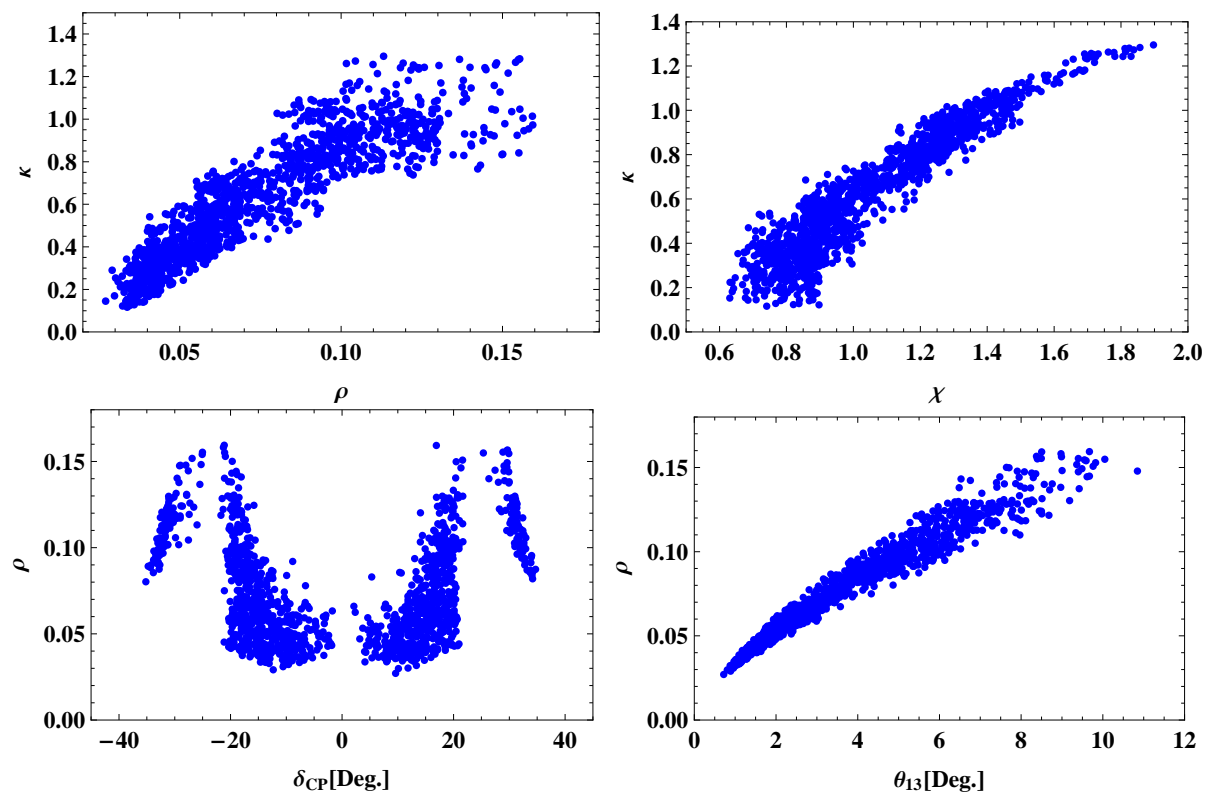

Fig. 4. The allowed parameter $\kappa, \rho, \chi$ (top panels) and the predictions of the Dirac $\mathrm{CP}$ violating phase $\delta_{\mathrm{CP}}$ (bottom-left panel) and the mixing angle $\theta_{13}$ (bottom-right panel) of the pattern $\mathrm{B}_{2}$.
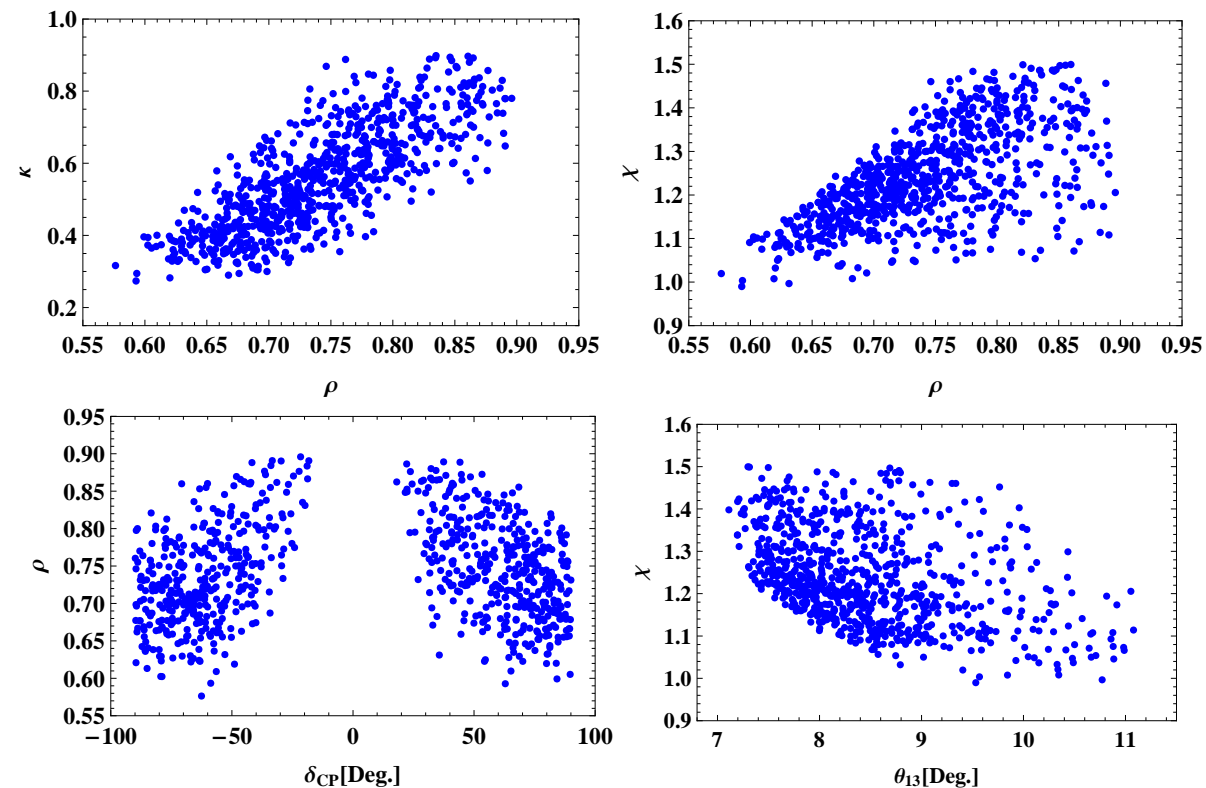

Fig. 5. The allowed parameter $\kappa, \rho, \chi$ (top panels) and the predictions of the Dirac $\mathrm{CP}$ violating phase $\delta_{\mathrm{CP}}$ (bottom-left panel) and the mixing angle $\theta_{13}$ (bottom-right panel) of the pattern $\mathrm{B}_{3}$. 

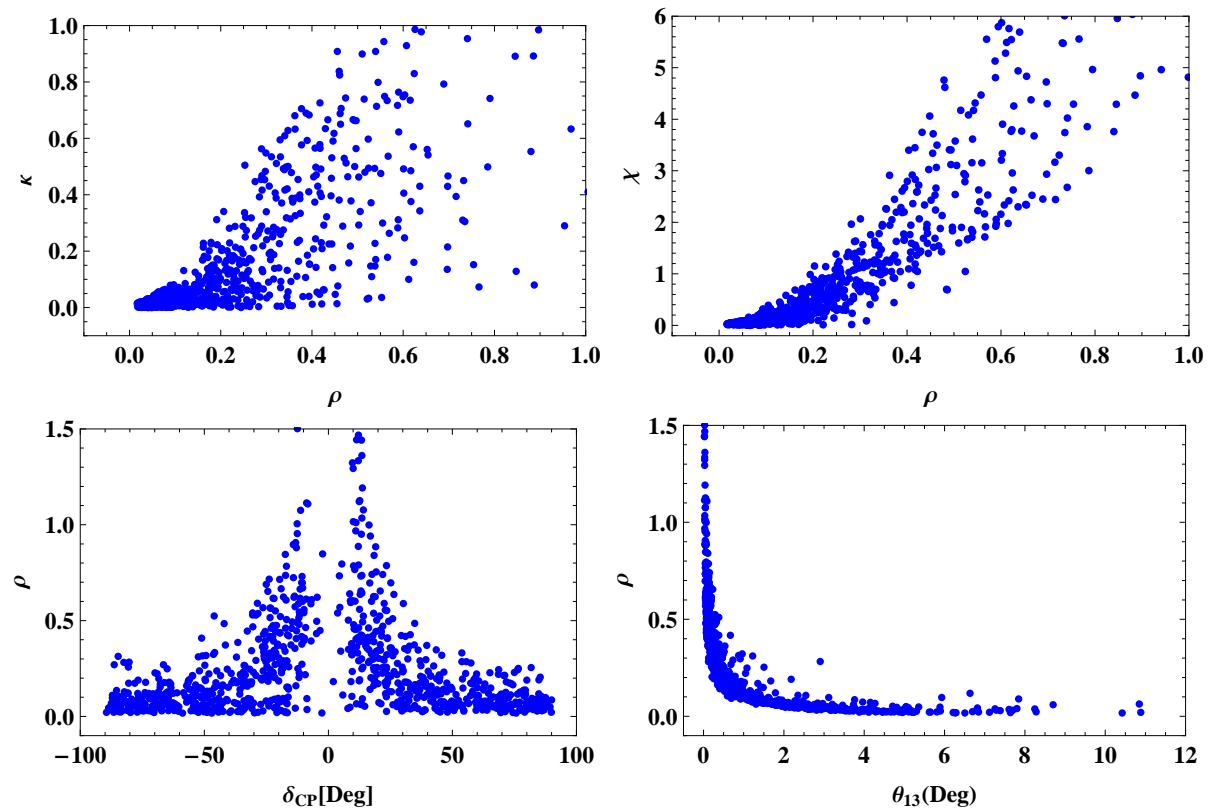

Fig. 6. The allowed parameter $\kappa, \rho, \chi$ (top panels) and the predictions of the Dirac $\mathrm{CP}$ violating phase $\delta_{\mathrm{CP}}$ (bottom-left panel) and the mixing angle $\theta_{13}$ (bottom-right panel) of the pattern $\mathrm{B}_{4}$.
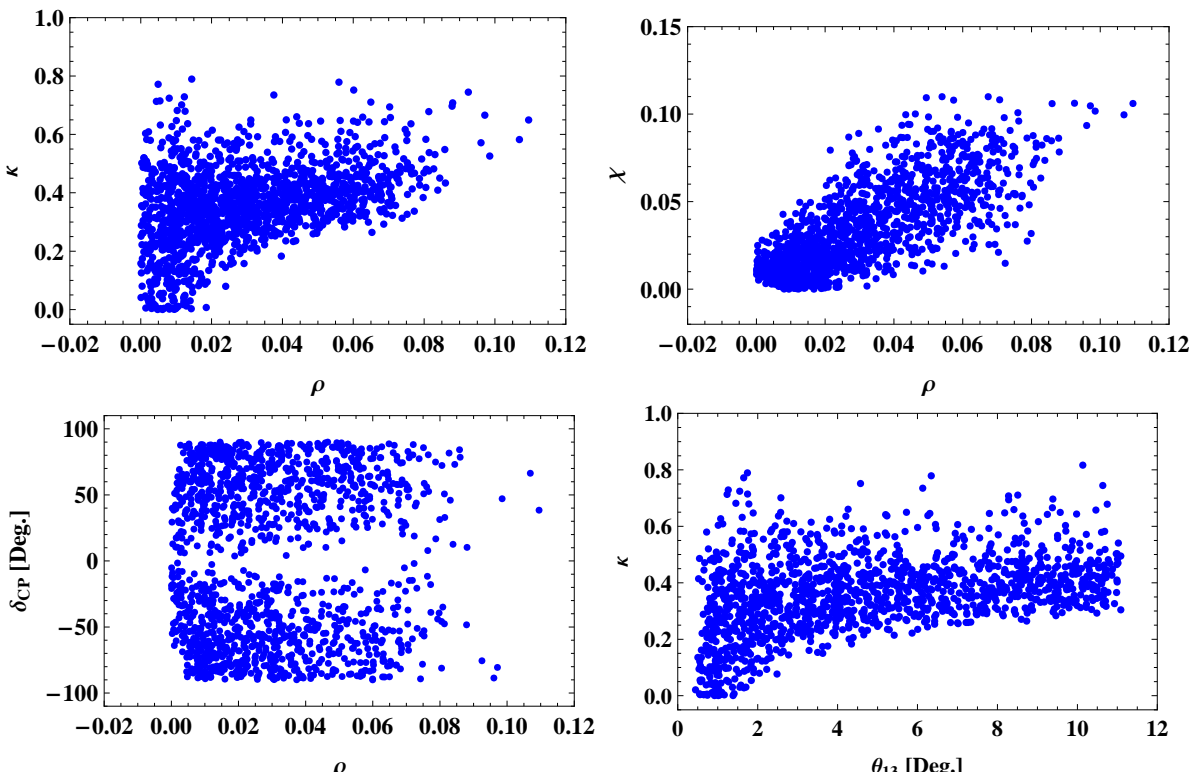

Fig. 7. The allowed parameter $\kappa, \rho, \chi$ (top panels) and the predictions of the Dirac $\mathrm{CP}$ violating phase $\delta_{\mathrm{CP}}$ (bottom-left panel) and the mixing angle $\theta_{13}$ (bottom-right panel) of the pattern $\mathrm{C}$.

We can see that, the prediction of the Dirac $\mathrm{CP}$ violating phase for the pattern $\mathrm{B}_{2}$ lie in the range $-40^{0} \rightarrow 40^{\circ}$, whereas for all the other patterns this phase has the range 
$-90^{0} \rightarrow 90^{0}$. Concern with the mixing angle $\theta_{13}$, the prediction of the pattern $\mathrm{B}_{1}$ for this mixing angle is small, $\theta_{13}<1^{0}$, so that this pattern could be excluded. The predictions of $\theta_{13}$ of the remain textures are agreed with the present experimental data. It is worth to remark here that we just study with the normal hierarchy of neutrino mass. For the inverted case we can examine in the similar way.

\section{CONCLUSIONS}

Under the latest results of neutrino oscillation experiments after neutrino - 2012 conference which indicate the relative large of the mixing angle $\theta_{13}$, we re-study the seven neutrino mass matrices with two independent vanishing entries. We first diagonalize the Hermitian matrix $m_{\nu}^{\dagger} m_{\nu}$ to find the low energy observables (squared neutrino masses, mixing angles and the Dirac CP violating phase) in terms of the parameters appeared in the neutrino mass matrix. By imposing the current experimental data for the normal spectrum of neutrino mass on those low energy observables, we scan for the allowed parameter spaces of the neutrino mass matrices and also give the predictions for the mixing angle $\theta_{13}$ and the Dirac CP violating phase. It turns out that the pattern $\mathrm{B}_{1}$ could be excluded because the angle $\theta_{13}$ is very small in this texture. Besides, we find that all the seven patterns possess the normal hierarchy of neutrino mass which is different with the reference [5] where only four patterns $\left(\mathrm{A}_{1}, \mathrm{~A}_{2}, \mathrm{~B}_{1}\right.$ and $\left.\mathrm{B}_{3}\right)$ have the normal hierarchy of neutrino mass spectrum.

\section{ACKNOWLEDGMENTS}

This work is funded by Scientific Research Foundation of Can Tho University under grant number T2013-23.

\section{REFERENCES}

[1] Super-Kamiokande Collaboration, Y. Fukuda et al., Phys. Rev. Lett. 81 (1998) 1562;

[2] Super-Kamiokande Collaboration, Y. Fukuda et al., Phys. Rev. Lett. 82 (1999) 2644.

[3] Super-Kamiokande Collaboration, Y. Fukuda et al., Phys. Rev. Lett. 82 (1999) 5194.

[4] P. H. Frampton, S. L. Glashow and D. Marfatia, Phys. Lett. B 536 (2002) 79.

[5] Z. Z. Xing, Phys. Lett. B 530 (2002) 159.

[6] Z. Z. Xing, Phys. Lett. B 539 (2002) 85.

[7] A. Kageyama, S. Kaneko, N. Simoyama and M. Tanimoto, Phys. Lett. B 538 (2002) 96.

[8] H. Fritzsch et al. JHEP 1109 (2011) 083 [arXiv:hep-ph/1108.4534].

[9] J. Y. Liu, S. Zhou, Phys.Rev. D 87 (2013) 093010 [arXiv:hep-ph/1304.2334].

[10] D.V. Forero et al., Phys.Rev. D 86 (2012) 073012 [arXiv:hep-ph/1205.4018].

[11] T2K Collaboration, K. Abe et al., Phys. Rew. Lett. 107 (2011) 041801

[12] MINOS Collaboration, P. Adamson et al., Phys. Rew. Lett. 107 (2011) 181802

[13] RENO Collaboration, J. Ahn et al., Phys. Rev. Lett. 108 (2012) 191802 [arXiv:hep-ex/1204.0626].

[14] Double CHOOZE Collaboration, Y. Abe et al., Phys. Rew. Lett. 108 (2012) 231081

[15] Daya Bay Collaboration, F. An et al., Phys. Rew. Lett. 108 (2012) 171803

[16] I. Aizawa, M. Yasue, Phys.Lett. B 607 (2005) 267-275 [arXiv:hep-ph/04009331] 